\title{
Axial Length and Ocular Development of Premature Infants without ROP
}

\author{
Yi Zha, Guangdong Zhu, Jinfei Zhuang, Haihua Zheng, Jianqiu Cai, and Wangqiang Feng
}

The 2nd Affiliated Hospital and Yuying Children's Hospital of Wenzhou Medical University, 109 Xueyuan Road, Wenzhou, Zhejiang 325027, China

Correspondence should be addressed to Wangqiang Feng; cy@wzhealth.com

Received 1 March 2017; Revised 24 July 2017; Accepted 17 August 2017; Published 15 October 2017

Academic Editor: Rune Brautaset

Copyright ( 2017 Yi Zha et al. This is an open access article distributed under the Creative Commons Attribution License, which permits unrestricted use, distribution, and reproduction in any medium, provided the original work is properly cited.

\begin{abstract}
Purpose. To investigate the ocular parameters of premature infants without ROP at gestational age (GA) more than 28 weeks and their relationship with growth parameters. Methods. 76 preterm infants without ROP and 65 term infants were involved to undergo portable slit lamp, RetCam3, ultrasonic A-scan biometry, and cycloplegic streak examination at their 40 weeks' postconceptional ages (PCA). Ocular parameters of infants' right eye and growth parameters were used for analysis. Results. All the infants were examined at 40 weeks' PCA. No significant difference was found between male and female in axial length of preterm infants $(p=0.993)$ and term infants $(p=0.591)$. Significant differences were found in axial length (AL), anterior chamber depth (ACD), lens thickness (LT), and vitreous depth (VD) between preterm and term infants. No significant correlation was found between $\mathrm{AL}$ and spherical equivalent in preterm infants' group. In preterm group, AL was significantly correlated with gestational age (GA), birth weight (BW), and head circumference (HC). Conclusions. Preterm infants had shorter AL, shallow ACD, thicker LT, and thinner VD compared to term infants. Refractive error in preterm infants at GA between 28 to 37 weeks was not related to axial length. Among all the growth parameters of preterm infants, GA, BW, and HC had effect on axial length.
\end{abstract}

\section{Introduction}

Due to the rapid prevalence of myopia, refractive error has long been the most important eye problem throughout the world especially in Asian countries [1]. Prematurity, with childbirth before 37 weeks of pregnancy, is associated with many ocular abnormalities, such as retinopathy of prematurity (ROP), refractive error, and amblyopia. Refractive error, on the other hand, is found to be related to not only prematurity but also increasing severity of retinopathy of prematurity (ROP) as well [2]. The increasing risk of refractive error in prematurely born infants is nowadays urgent to be solved.

The major proportion of eye growth occurs within the first 12 months after birth [3]. It is well known that term infants are commonly hypermetropic, while preterm infants are always associated with myopia [4-7]. Many researchers have found that the development of myopia in premature infants may be related to ocular parameters, such as axial length and anterior chamber depth $[6,8,9]$. Others tended to believe its association with the corneal curvature and refractive power of the lens [10-12]. Current studies focused more on premature infants with gestational age (GA) lower than 28 weeks, who were apt to suffer from ROP. In the present study, we investigated the ocular parameters of premature infants with GA more than 28 weeks and their relationship with growth parameters.

\section{Materials and Methods}

All the infants involved were recruited from the Department of Neonatology of the 2nd Affiliated Hospital of Wenzhou Medical University. Regional ethics committee approval and parental consent were obtained. Exclusion criteria included ocular anomalies such as microphthalmos, anophthalmos, craniofacial deformities, congenital glaucoma, cornea, and lens, or any other fundus abnormalities (such as ROP) and a history of cerebral damage. Premature infants with GA of less than 28 weeks were also excluded in the study. There was no geographic or ethnic dissimilarity in this study. All the infants were Chinese. 
At the first visit, growth parameters such as GA, length, birth weight (BW), and head circumference (HC) at birth were obtained. At 40 weeks' postconceptional ages (PCA) (defined as the gestational age at birth plus the age in weeks at the time of examination), all the infants including premature and term infants underwent portable slit lamp, RetCam3 (Clarity Medical System, USA), ultrasonic A-scan biometry, and cycloplegic streak retinoscopy (cyclopentolate $0.5 \%$ ) instilled at 10-minute intervals three times, 40 minutes before retinoscopy. RetCam3 was used to exclude ROP or other retinal disease. Cycloplegic streak retinoscopy was used to get the refractive status of each infant. The ultrasonic examination was performed using an A-scan biometer (Carl Zeiss Meditec, Oberkochen, Germany) to measure the axial length $(\mathrm{AL})$, anterior chamber depth (ACD), lens thickness (LT), and vitreous depth (VD). The probe was placed lightly on the center of the cornea, perpendicular to its axis. The probe was maintained in this position until three clear traces were obtained on the screen. The average value from the three images was recorded.

All the data obtained were analyzed using SPSS (version 17.0; SPSS Inc., Chicago, IL, USA). The values obtained for all parameters were expressed as mean \pm SD. $t$-test was used to compare parameters between two groups. Simple linear regression analysis was performed to get the association of axial length with each of the variables, namely, spherical equivalent (SE), GA, length, BW, and HC. A $p$ value below 0.05 was considered significant.

\section{Results}

76 premature infants (with 27 female and 49 male) and 65 term infants (with 32 female and 33 male) were involved in the research. The data of infants' right eye were chosen for analysis. The GA in the preterm group was $32.97 \pm 2.15 \mathrm{w}$ and in the term group, $39.29 \pm 1.30 \mathrm{w}$. All the growth parameters for preterm and term infants were shown in Table 1. No significant difference was found between male and female in axial length of preterm infants $(p=0.993)$ and term infants $(p=0.591)$. The mean and SD of infants' SE, AL, ACD, LT, and VD were shown in Table 2. Significant differences were found in $\mathrm{AL}, \mathrm{ACD}, \mathrm{LT}$, and $\mathrm{VD}$ between preterm and term infants' groups.

In preterm group, $\mathrm{AL}$ was significantly correlated with GA $(r=0.312, p=0.006)$, BW $(r=0.344, p=0.002)$, and $\mathrm{HC}(r=0.241, p=0.041)$ but not correlated with SE $(r=-0.226, p=0.05)$ and length $(r=0.229, p=0.053)$.

\section{Discussion}

This research focused on Chinese premature infants born at GA between 28 to 37 weeks without ROP or other ocular disease and evaluated the association of AL with other ocular and growth parameters. Our study showed that with a GA of 28 to 37 weeks, premature infants did have lower AL, shallow ACD, thicker lens, and shorter VD (Table 2). AL was significantly correlated with GA, BW, and HC.

Fieß A et al. [13] once compared the axial length and anterior segment alterations in preterm infants with those
TABLE 1: Growth parameters and age for both preterm and term infants.

\begin{tabular}{lcc}
\hline Growth parameters & $\begin{array}{c}\text { Preterm infants } \\
(\text { mean } \pm \text { SD })\end{array}$ & $\begin{array}{c}\text { Term infants } \\
(\text { mean } \pm \text { SD) }\end{array}$ \\
\hline Gestational age (weeks) & $32.97 \pm 2.15$ & $39.29 \pm 1.30$ \\
Birth weight $(\mathrm{g})$ & $1930 \pm 525$ & $3232 \pm 475$ \\
Head circum. $(\mathrm{cm})$ & $30.68 \pm 1.98$ & $33.93 \pm 1.35$ \\
Length $(\mathrm{cm})$ & $42.83 \pm 4.38$ & $49.98 \pm 2.50$ \\
\hline
\end{tabular}

of full-term infants and found significant differences between preterm and full-term infants aged $\leq 7$ years for spherical equivalent, astigmatism, corneal diameter, and axial length. Tian et al. [14] investigated the development of the refractive status in premature infants aged 0 to 6 years old and found that the axial length in preterm infants was significant shorter than that in term infants. Ecsedy et al. [15] compared the ocular geometry and refraction in children with a history of preterm birth and found that in the premature eyes, anterior chamber depth was marginally smaller, the lens was significantly thicker, and axial length was significantly shorter. All the research above focused on older preterm children. In our research, we measured the ocular parameters in preterm infants with GA between 28 and 37 weeks without ROP and found that preterm infant had shorter AL, smaller ACD, thicker lens, and shorter VD. As we know, ocular structures go through a continuous development and remodeling process after birth [16]. Premature departure from the intrauterine environment may affect ocular development [17]. We believe that premature birth even with GA older than 28 weeks delays the development of ocular structures, which happens sooner after birth.

Preterm infants were thought to be more myopic than term infants [8]. However, ocular biometry and the mechanism of myopia in preterm children were somewhat different from those in full-term children. Anterior segment components were thought to contribute more to myopia progression in preterm children $[8,18-20]$. Axial length, on the other hand, changed differently [18-21]. According to our result, axial length had no significant relationship with spherical equivalent. It was considered that early refractive errors may not provide enough information in predicting the later refractive and axial length outcome.

Fieß A et al. [13] detected axial length associated with birth weight and age. Modrzejewska et al. [9] found a correlation between axial length and birth weight. In our research, AL was significantly correlated with GA, BW, and HC. As the major proportion of eye growth occurs within the first year after birth, our observation suggested that ocular growth be affected by prematurity and led to shorter axial length, especially in the first years of life.

The prematurely born children had a higher prevalence of hypermetropia ( $>3 \mathrm{D}$ ) and clinically significant myopia $(\leq-1 \mathrm{D})$ than those born at term. Moderate or high myopia $(<-3 \mathrm{D})$ was found only in preterm group. In our preterm group, take the right eye for granted, there were $22.37 \%$ (17/76) of hypermetropia (>3 D) and $15.78 \%$ (12/76) of significant myopia $(\leq-1 \mathrm{D})$. Four infants had moderate or high 
TABLE 2: Parameter analysis of variance between preterm and term infants at 40 weeks' PCA.

\begin{tabular}{lccc}
\hline Parameter for right eye & Term infants $($ mean \pm SD) & Preterm infants $($ mean \pm SD) & $p$ \\
\hline Number & 65 & 76 & \\
Spherical equivalent (SE, D) & $2.19 \pm 2.22$ & $1.96 \pm 2.12$ & 0.50 \\
Axial length (AL, mm) & $17.34 \pm 0.55$ & $17.08 \pm 0.67$ & ${ }^{*} \mathbf{0 . 0 2}$ \\
Anterior chamber depth (ACD, mm) & $2.55 \pm 0.26$ & $2.38 \pm 0.25$ & ${ }^{*} \mathbf{0 . 0 0 0}$ \\
Lens thickness (LT, mm) & $3.72 \pm 0.18$ & $3.99 \pm 0.15$ & $* \mathbf{0 . 0 0 0}$ \\
Vitreous depth (VD, cm) & $11.03 \pm 0.46$ & $10.79 \pm 0.56$ & $* \mathbf{0 . 0 1}$ \\
\hline
\end{tabular}

${ }^{*} p<0.05$ significant difference between two groups.

myopia $(\leq-3 \mathrm{D})$. The percentages of hypermetropia and significant myopia were higher than those of our term group (with 10/65 of hypermetropia and 5/65 of significant myopia). Due to the limitation of sample size, further studies were needed for larger sample investigation.

In this research, we investigated the axial length and refractive status of preterm infants with a GA of more than 28 weeks without ROP and found that preterm infants had shorter AL, shallow ACD, thicker LT, and thinner VD. Axial length in preterm infants was significantly correlated with gestational age, birth weight, and head circumference.

\section{Conflicts of Interest}

The authors declare that there is no conflict of interest regarding the publication of this paper.

\section{Acknowledgments}

The authors thank Professor Mingguang Shi for his helpful comments and suggestions.

\section{References}

[1] P. J. Foster and Y. Jiang, "Epidemiology of myopia," Eye, vol. 28, no. 2, pp. 202-208, 2014.

[2] A. Moskowitz, R. M. Hansen, and A. B. Fulton, "Retinal, visual, and refractive development in retinopathy of prematurity," Eye Brain, vol. 8, pp. 103-111, 2016.

[3] M. E. Hartnett and J. S. Penn, "Mechanisms and management of retinopathy of prematurity," The New England Journal of Medicine, vol. 367, no. 26, pp. 2515-2526, 2012.

[4] A. R. O'Connor, C. M. Wilson, and A. R. Fielder, "Ophthalmological problems associated with preterm birth," Eye, vol. 21, no. 10, pp. 1254-1260, 2007.

[5] R. M. Varghese, V. Sreenivas, J. M. Puliyel, and S. Varughese, "Refractive status at birth: its relation to newborn physical parameters at birth and gestational age," PLoS One, vol. 4, no. 2, article e4469, 2009.

[6] S. Bhatti, E. A. Paysse, M. P. Weikert, and L. Kong, "Evaluation of structural contributors in myopic eyes of preterm and fullterm children," Graefe's Archive for Clinical and Experimental Ophthalmology, vol. 254, no. 5, pp. 957-962, 2016.

[7] H. C. Fledelius, R. Bangsgaard, C. Slidsborg, and M. laCour, "Refraction and visual acuity in a national Danish cohort of 4 -year-old children of extremely preterm delivery," Acta Ophthalmologica, vol. 93, no. 4, pp. 330-338, 2015.
[8] T. C. Chen, T. H. Tsai, Y. F. Shih et al., "Long-term evaluation of refractive status and optical components in eyes of children born prematurely," Investigative Ophthalmology \& Visual Science, vol. 51, no. 12, pp. 6140-6148, 2010.

[9] M. Modrzejewska, W. Grzesiak, D. Karczewicz, and D. Zaborski, "Refractive status and ocular axial length in preterm infants without retinopathy of prematurity with regard to birth weight and gestational age," Journal of Perinatal Medicine, vol. 38, no. 3, pp. 327-331, 2010.

[10] C. S. Yang, A. G. Wang, Y. F. Shih, and W. M. Hsu, "Astigmatism and biometric optic components of diode laser-treated threshold retinopathy of prematurity at 9 years of age," Eye, vol. 27, no. 3, pp. 374-381, 2013.

[11] M. Snir, R. Friling, D. Weinberger, I. Sherf, and R. Axer-Siegel, "Refraction and keratometry in 40 week old premature (corrected age) and term infants," The British Journal of Ophthalmology, vol. 88, no. 7, pp. 900-904, 2004.

[12] C. S. Yang, A. G. Wang, Y. F. Shih, and W. M. Hsu, "Longterm biometric optic components of diode laser-treated threshold retinopathy of prematurity at 9 years of age," Acta Ophthalmologica, vol. 91, no. 4, pp. e276-e282, 2013.

[13] A. Fieß, R. Kölb-Keer, M. Knuf et al., "Axial length and anterior segment alterations in former preterm infants and full-term neonates analyzed with Scheimpflug imaging," Cornea, vol. 36, no. 7, pp. 821-827, 2017.

[14] M. Tian, L. Zhou, Q. Luo, Y. M1, and Y. Xu, "Study of refractive state in premature infants without retinopathy of prematurity and full-term children at the age of 0 to 6," Zhonghua Yan Ke Za Zhi, vol. 51, no. 7, pp. 505-509, 2015.

[15] M. Ecsedy, I. Kovacs, K. Mihaltz et al., "Scheimpflug imaging for long-term evaluation of optical components in Hungarian children with a history of preterm birth," Journal of Pediatric Ophthalmology and Strabismus, vol. 51, no. 4, pp. 235-241, 2014.

[16] D. J. De Silva, K. D. Cocker, G. Lau, S. T. Clay, A. R. Fielder, and M. J. Moseley, "Optic disk size and optic disk-to-fovea distance in preterm and full-term infants," Investigative Ophthalmology \& Visual Science, vol. 47, no. 11, pp. 4683-4686, 2006.

[17] K. J. Saunders, D. L. McCulloch, A. J. Shepherd, and A. G. Wilkinson, "Emmetropisation following preterm birth," The British Journal of Ophthalmology, vol. 86, no. 9, pp. 10351040, 2002.

[18] A. Cook, S. White, M. Batterbury, and D. Clark, "Ocular growth and refractive error development in premature infants with or without retinopathy of prematurity," Investigative Ophthalmology \& Visual Science, vol. 49, no. 12, pp. 51995207, 2008. 
[19] A. R. O’Connor, T. J. Stephenson, A. Johnson, M. J. Tobin, S. Ratib, and A. R. Fielder, "Change of refractive state and eye size in children of birth weight less than $1701 \mathrm{~g}$," The British Journal of Ophthalmology, vol. 90, no. 4, pp. 456-460, 2006.

[20] A. R. O'Connor, C. M. Wilson, and A. R. Fielder, "Ophthalmological problems associated with preterm birth," Eye, vol. 21, no. 10, pp. 1254-1260, 2007.

[21] M. Kaya, A. T. Berk, and A. Yaman, "Long-term evaluation of refractive changes in eyes of preterm children: a 6-year followup study," International Ophthalmology, pp. 1-8, 2017. 


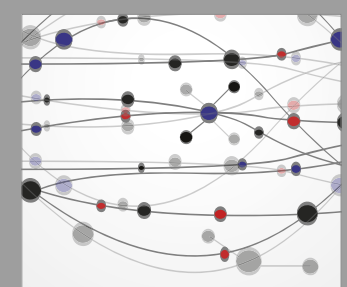

The Scientific World Journal
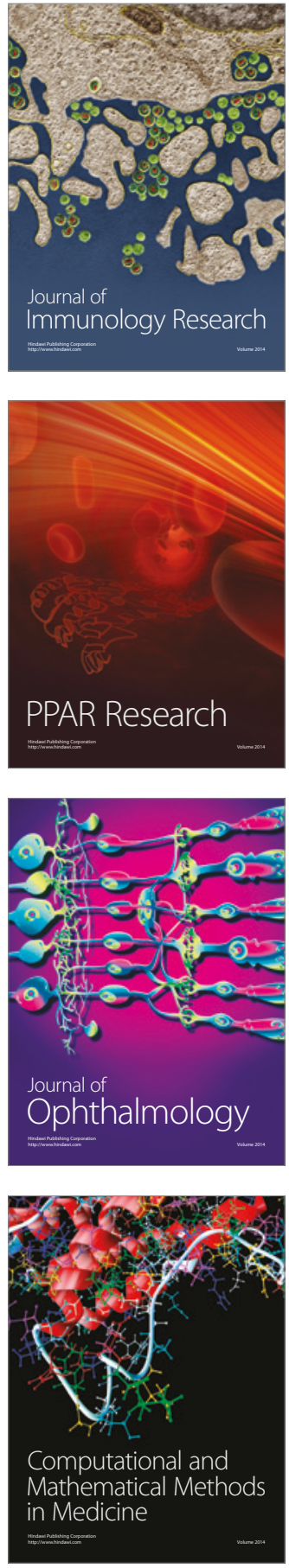

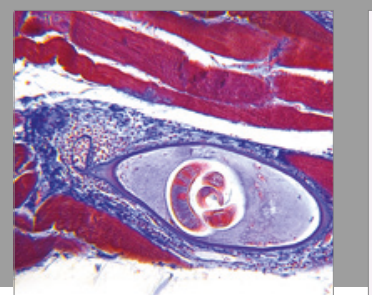

Gastroenterology Research and Practice
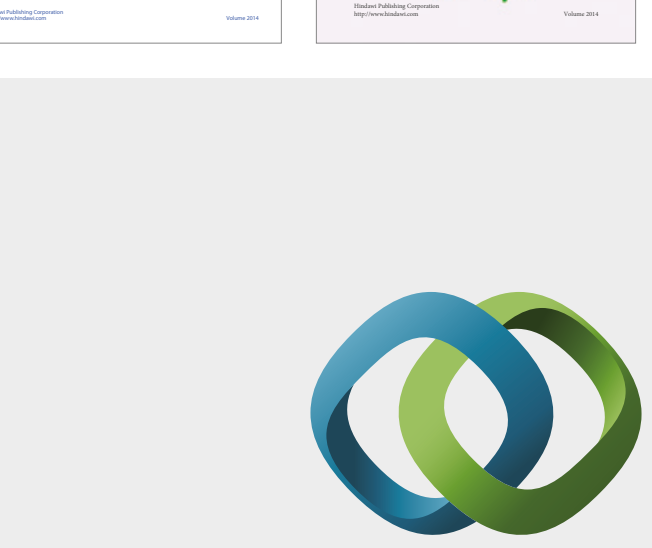

\section{Hindawi}

Submit your manuscripts at

https://www.hindawi.com
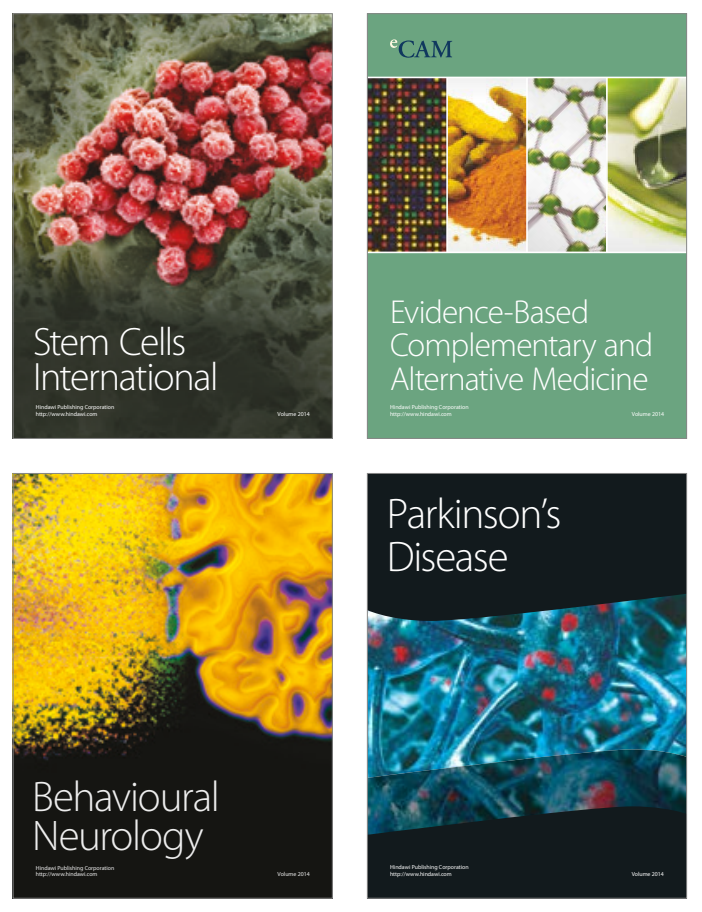
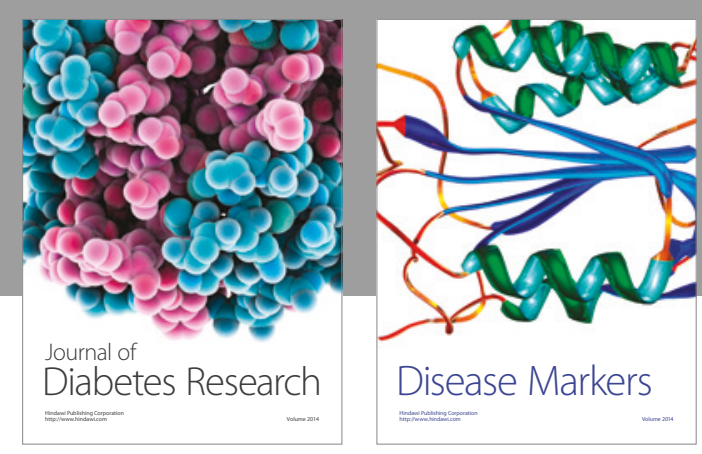

Disease Markers
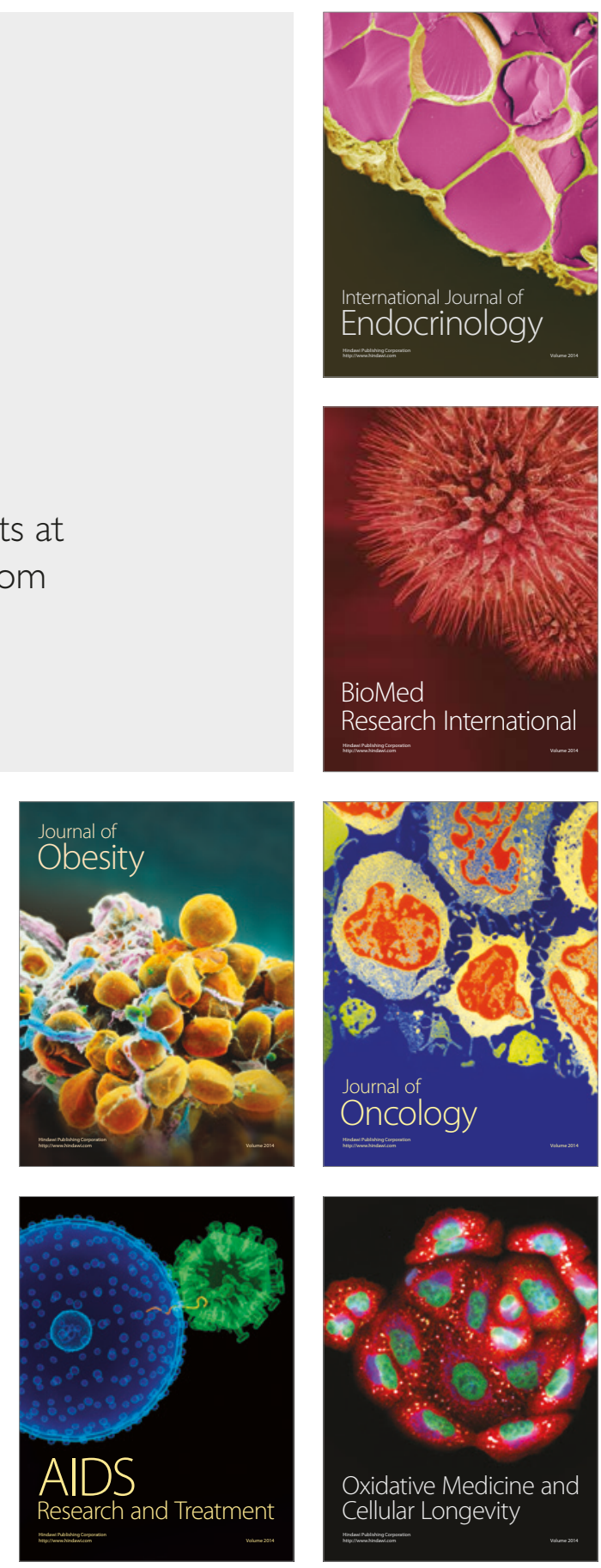